\title{
Morbidity and Mortality Patterns in Children Admitted to Hospital in Thai Binh, Vietnam: A Five-year Descriptive Study with a Focus on Infectious Diseases
}

\author{
Thi Dung Pham ${ }^{1,+,}, \mathbb{D}$, Van Thuan Hoang ${ }^{1,2,3, t,(\mathbb{D}}$, Thi Loi Dao ${ }^{1,2,3,}, \mathbb{D}$, Xuan Duong Tran $^{1}$, Duc Long Phi ${ }^{1}$, \\ Minh Manh To ${ }^{1}$, Van Nghiem Dang ${ }^{1}$, Van Khoi Dang ${ }^{1}$, Thanh Tung Dao ${ }^{4}$, Nam Thang Nguyen ${ }^{1}$, Thi Thuy Vu ${ }^{5}$, \\ Duc Thanh Nguyen ${ }^{1}$, Duy Cuong Nguyen ${ }^{1}$, Nang Trong Hoang ${ }^{1}$, Thanh Liem Vu ${ }^{5}$, Thi Minh Chinh Nguyen ${ }^{5}$, \\ Philippe Minodier ${ }^{3,6,7}$, Philippe Gautret ${ }^{2,3,}$, (i)
}

${ }^{1}$ Thai Binh University of Medicine and Pharmacy, Thai Binh, Vietnam ${ }^{2}$ Aix Marseille Univ, IRD, AP-HM, SSA, VITROME, Marseille, France ${ }^{3}$ IHU-Méditerranée Infection, Marseille, France

${ }^{4}$ Thanh Hoa province Food safety Administration, Thanh Hoa, Vietnam

${ }^{5}$ Thai Binh Paediatric Hospital, Thai Binh, Vietnam

${ }^{6}$ Department of Paediatric Emergency, Centre Hospitalier Universitaire Nord, Marseille, France

${ }^{7}$ Groupe de Pathologie Infectieuse Pédiatrique, Paris, France

\section{ARTICLE INFO}

Article History

Received 05 May 2020

Accepted 11 July 2020

Keywords

Children

infectious disease

lower respiratory tract infections

gastrointestinal infections

influenza

Thai Binh

\begin{abstract}
The objective of this study was to describe the overall pattern of morbidity and mortality of children seen at the Thai Binh Paediatric Hospital in Vietnam, with a focus on infectious diseases. A retrospective review of hospitalisation records was conducted from 1 January 2015 to 31 December 2019. Data were obtained from a total of 113,999 records. The median age of patients was 18 months, with $84.0 \%$ of patients aged $<5$ years. Infectious diseases accounted for $61.0 \%$ of all cases. The most prevalent diseases were lower respiratory tract infections (32.8\%), followed by gastrointestinal infections (13.3\%) and confirmed influenza (5.4\%). Most infections were not microbiologically documented. A total of $81.4 \%$ patients received at least one antibiotic. Most patients (97.0\%) were hospitalised for less than 15 days. Regarding outcomes, $87.8 \%$ patients were discharged home with a favourable outcome. Twelve percent were transferred to the Vietnam National Children's Hospital because their condition had worsened and $0.1 \%$ died. In total, infectious diseases accounted for $40.4 \%$ of deaths, followed by neonatal disorders (34.6\%). Our data serves a basis for the identification of needs for diagnostic tools and for future evaluation of the effect of the targeted implementation of such facilities. Point-of-care tests, including real-time polymerase chain reaction assays to identify common pathogens should be implemented for more accurate diagnosis and more appropriate antibiotic use.
\end{abstract}

(C) 2020 The Authors. Published by Atlantis Press International B.V.

This is an open access article distributed under the CC BY-NC 4.0 license (http://creativecommons.org/licenses/by-nc/4.0/).

\section{INTRODUCTION}

Across most low-income countries, levels of both child and maternal mortality have dropped over the past three decades. The United Nations International Children's Emergency Fund Millennium Development Goals succeeded in reducing child mortality by almost half around the world between 1990 and 2018 [1]. This has led to changes in the patterns of diseases which cause mortality in under-fives.

Preventable diseases such as Respiratory Tract Infections (RTIs), diarrhoeal diseases, malaria and Human Immunodeficiency Virus (HIV) infection are major causes of childhood morbidity and

"Corresponding author.Email:philippe.gautret@club-internet.fr "Equal work.

Data availability statement: The data that support the findings of this study are available from the corresponding author, [PG], upon reasonable request. mortality world-wide [1]. The World Health Organization estimated that in 2018, 6.2 million children and adolescents under the age of 15 die annually, mostly from preventable causes. Of these deaths, over 5 million occurred in the child's first 5 years, with almost half of these in the first month of life [1]. These diseases can be prevented, and treatments are accessible and affordable [1]. For children who are five and over, non-communicable diseases were the most common causes of death [2].

In Vietnam, the under-five mortality rate in 2010 was 19.4 per 1000 live births [3]. According to the Institute for Health Metrics and Evaluation, the major causes of child mortality in Vietnam in 2017 were maternal and neonatal disorders (31.3\%), congenital birth defects (26.6\%), Lower RTI (LRTI) (13.0\%), whooping cough (3.0\%), encephalitis $(2.3 \%)$, meningitis (1.3\%), diarrhoea $(0.7 \%)$ and $\mathrm{HIV}(0.3 \%)$ [4]. Antibiotic resistance is now emerging as a risk factor for mortality and prolonged hospital stay among Vietnamese children $[5,6]$. 
The objective of this study was to describe the overall pattern of morbidity and mortality of patients below the age of 16 and seen at the Thai Binh Paediatric Hospital, a referral hospital in the central province of Thai Binh. In this paper, we focus on Infectious Diseases (ID). These data will help in designing standardised protocols for the proper management of the most common infectious diseases presenting at this hospital. They will also serve for identifying gaps in diagnostic and management tools and for evaluating the future implementation of targeted policies.

\section{MATERIALS AND METHODS}

\subsection{Study Setting}

Thai Binh $\left(20^{\circ} 30^{\prime} \mathrm{N}, 106^{\circ} 20^{\prime} \mathrm{E}\right)$ is a coastal eastern province in the Red River Delta region of northern Vietnam. It is about $110 \mathrm{~km}$ from the capital city, Hanoi (Figure 1). In 2019, this province covers an area of $1542 \mathrm{~km}^{2}$ with $1,860,447$ people [7,8], mostly living in rural areas $(89.4 \%)$ with $15.5 \%$ of the population aged $0-9$ years and $12.8 \%$ aged $10-19$ years [8].

The Thai Binh Paediatric Hospital is a first-class hospital in the central province of Thai Binh (Figure 1). In 2019, this hospital had 565 beds spread across 15 different medical departments. In The number of beds in this hospital hardly meets the number of hospitalized patients. With the exception of the neonatal department, most other departments have an overload of patients compared to the number of existing beds. Eighty-six paediatricians and 171 nurses are currently working in the facility (Figure 1). Microbiological identification in the hospital laboratory uses common bacteriological cultures, serological assays and realtime Polymerase Chain Reaction (PCR) for a limited number of pathogens. Ill children under the age of 16 are directly admitted from home (through outpatient or emergency departments) or referred from eight district hospitals in the province of Thai Binh.
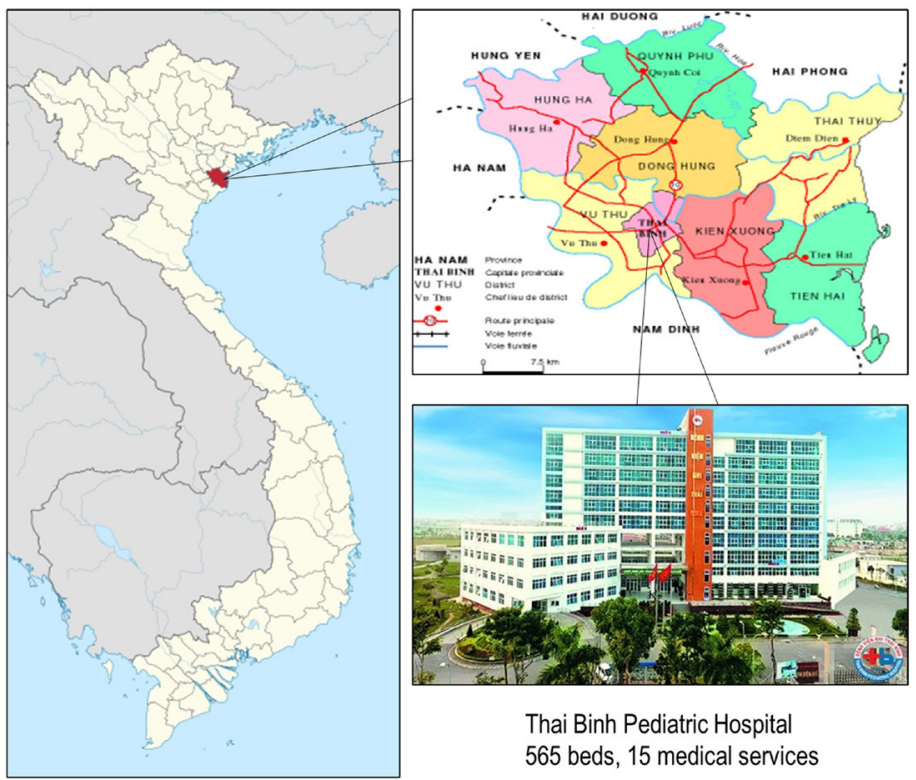

Thai Binh Pediatric Hospital 565 beds, 15 medical services
Children with severe diseases who do not improve, are referred to the Vietnam National Children's Hospital in Hanoi (VNCH). The medical records of patients have been digitally available only since June 2019, through a computerised system.

In Vietnam, all children under the age of 6 have free health insurance. Parents of children over the age of 6 then have to take out school-age health insurance. According to the expanded immunisation programme in Vietnam [9], tuberculosis vaccine is recommended as soon as possible within 30 days of birth. Three doses of pentavalent vaccine [diphtheria, tetanus, pertussis, hepatitis B and Haemophilus influenzae type B (Hib)] and oral polio vaccine are recommended between the ages of 2 and 4 months. The measles vaccine is recommended at 9-11 and 18 months. Three doses of Japanese encephalitis are recommended at 12 months, 2 weeks after the first dose and 1 year after the second dose. These vaccines are provided free of charge by the Vietnamese government. Vaccines against rotavirus and invasive pneumococcal diseases are optional, expensive and are not free of charge.

\subsection{Study Design and Population}

This is a retrospective review of consecutive morbidity and mortality occurring in children aged $0-16$ years admitted to the Thai Binh Paediatric Hospital from 1 January 2015 to 31 December 2019.

Ethical approval was obtained from the Ethics Committee of Thai Binh Paediatric Hospital (No. 2019-12.1).

\subsection{Data Collection Methods and Instruments}

All medical records of eligible subjects were collected from central computer of the hospital and analysed by a team of 12

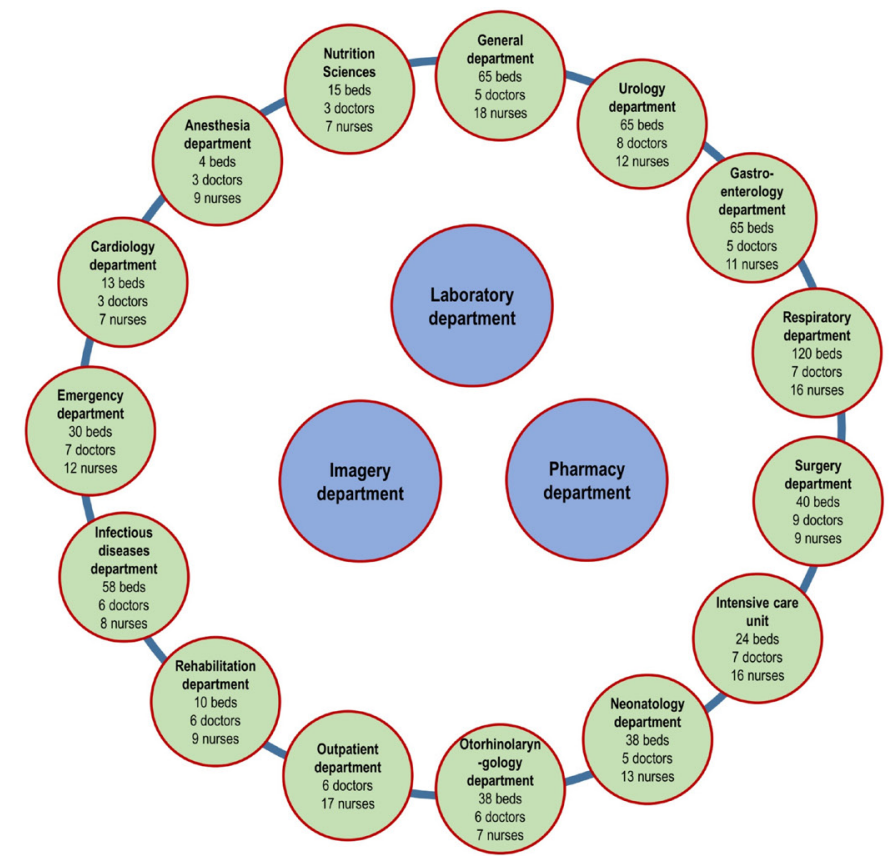

Figure 1 Study setting. 
medical doctors. The eligibility criteria were hospitalisation at the Thai Binh Paediatric Hospital, documentation of the date and month of admission, age, gender, diagnosis, treatment and outcome (defined as discharged home, transferred to the $\mathrm{VNCH}$, discharged against medical advice, or died).

Diagnoses at discharge were used for classification and categorised according to the International Classification of Diseases (ICD10 criteria) [10]. For concurrent diagnosis, children with more than one disease were grouped together depending on the doctor's primary and secondary diagnoses.

Data obtained were analysed using STATA software version 14.2 (Copyright 1985-2015 StataCorp LLC, http://www.stata.com). Continuous variables were analysed and expressed as median and Interquartile (IQR). Categorical variables were presented as percentages.

\section{RESULTS}

\subsection{Characteristics of the Study Population}

Data were obtained from a total of 113,999 hospitalisation records over the 5-year study period. The median age of patients was 18 months (interquartile range 6-42 months, $\min =0, \max =$ 16 years) with $84.0 \%(95,737 / 113,999)$ of patients aged $<5$ years. In total, $60.5 \%(69,016 / 113,999)$ individuals were male with a sex ratio $\mathrm{M} / \mathrm{F}$ of 1.5 . The age-gender pyramid is shown in Figure 2. $75.7 \%$ $(86,319 / 113,999)$ of patients lived in rural areas.

\subsection{Morbidity Patterns}

Table 1 shows the distribution of diseases by syndromes during the 5 -year study period. Overall, ID accounted for $61.0 \%$ of all cases. The most prevalent diseases were LRTI (32.8\%), gastrointestinal infections (13.3\%), and confirmed influenza (5.4\%). Other IDs included fever of unknown origin (3.7\%), skin infections (3.4\%), hand, foot and mouth disease (1.8\%), sepsis (1.3\%), viral hepatitis $(0.5 \%)$, meningitis/encephalitis $(0.2 \%)$, HIV or tuberculosis $(0.04 \%)$. Other infections represented $2.3 \%$ of the admissions. No cases of malaria were reported.

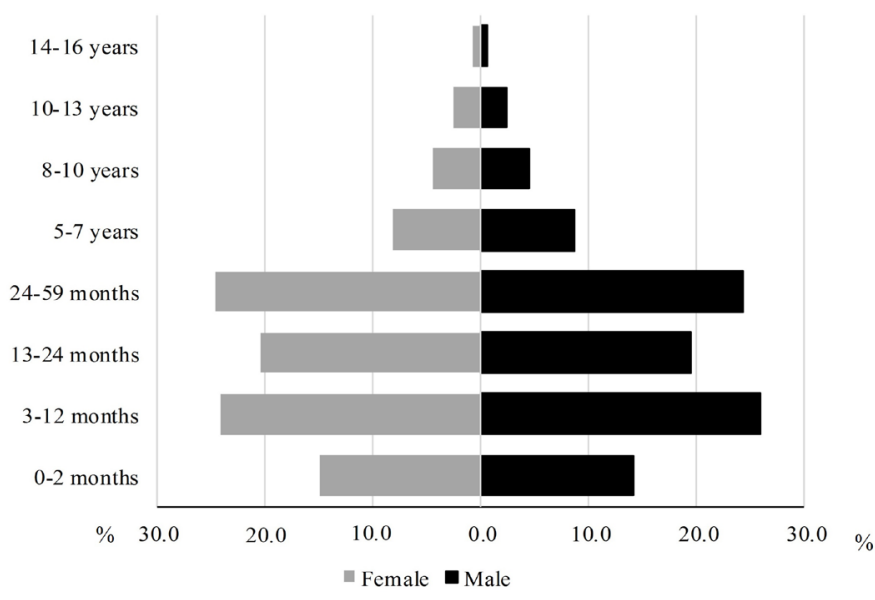

Figure 2 Age-gender pyramid of hospitalised patients.
Table 1 Relative proportion of diseases (diagnosis at discharge) according to syndromic classification and etiologic diagnoses according to available laboratory tests, in 113,999 hospitalisation records of children hospitalised at the Thai Binh Pediatric Hospital between 2015 and 2019

\begin{tabular}{|c|c|c|}
\hline Syndromes & $n$ & Percentage $(\%)$ \\
\hline Lower respiratory tract infections ${ }^{*}$ & 37,439 & 32.84 \\
\hline Gastrointestinal infections $^{*}$ & 15,175 & 13.31 \\
\hline Influenza* & 6132 & 5.38 \\
\hline Non-communicable gastrointestinal diseases & 5261 & 4.61 \\
\hline Other non-communicable diseases & 4471 & 3.92 \\
\hline Fever of unknown origin ${ }^{*}$ & 4196 & 3.68 \\
\hline Skin infection ${ }^{*}$ & 3875 & 3.38 \\
\hline Neonatal disorders & 3063 & 2.69 \\
\hline Other infections ${ }^{*}$ & 2631 & 2.31 \\
\hline Unintentional injuries & 2089 & 1.83 \\
\hline Hand, foot and mouth diseases ${ }^{*}$ & 2011 & 1.76 \\
\hline Haemoglobinopathies and haemolytic anaemia & 1618 & 1.42 \\
\hline Sepsis $^{*}$ & 1444 & 1.27 \\
\hline Congenital birth defects & 1414 & 1.24 \\
\hline Convulsion and epilepsy & 1095 & 0.96 \\
\hline Cardiovascular diseases & 969 & 0.85 \\
\hline Neurological disorders & 852 & 0.75 \\
\hline Diabetes and chronic kidney diseases & 785 & 0.69 \\
\hline Headaches & 782 & 0.69 \\
\hline Asthma and other chronic respiratory diseases & 658 & 0.58 \\
\hline Mental disorders & 655 & 0.57 \\
\hline Viral hepatitis ${ }^{*}, \underline{z}$ & 508 & 0.45 \\
\hline Nutritional deficiencies & 418 & 0.37 \\
\hline Food or drug poisoning & 318 & 0.28 \\
\hline Meningitis and encephalitis ${ }^{*}$ & 250 & 0.22 \\
\hline Cirrhosis and other chronic liver diseases & 168 & 0.15 \\
\hline Leukaemia & 114 & 0.10 \\
\hline HIV and tuberculosis* & 45 & 0.04 \\
\hline At least one infectious disease & 69,536 & 60.99 \\
\hline
\end{tabular}

"Infectious diseases. ${ }^{\mathrm{p}}$ Hepatitis B, C or cytomegalovirus.

Figure 3 shows the changes in morbidity patterns in hospitalised children in 2019 as compared to 2015. LRTI was consistently the most frequent cause of morbidity in hospitalised children. However, in 2019, influenza ranked as the second cause of hospitalisation, compared to ranking 25th in 2015. Gastrointestinal infections remained one of the most frequent causes of admission after respiratory infections over the study period.

A total of $81.4 \%(92,742 / 113,999)$ patients received at least one antibiotic, regardless of their diagnosis. Of them, 22.4\% (20, 744/92, 742) were treated with an antibiotic combination. Among all patients, the most frequent antibiotic used was cephalosporin $(65.6 \%)$, followed by penicillin (17.7\%), aminoglycoside (11.3\%), imidazole (3.8\%), macrolide (2.0\%), carbapenem $(1.5 \%)$ and quinolone $(1.0 \%)$. Other antibiotics were prescribed in $1.2 \%$ patients (data not shown).

Overall, the mean length of hospital stay was $6.0 \pm 5.2$ days $(\mathrm{IQR}=$ 3-7 days). Three percent of patients were hospitalised for more than 15 days.

\subsection{Outcomes and Mortality Pattern}

At total of $87.8 \%(100,043 / 113,999)$ patients were discharged home with a favourable outcome, $12 \%(13,680 / 113,999)$ were transferred to the $\mathrm{VNCH}$ because their condition had worsened, $0.1 \%$ 
2015

1. Lower respiratory tract infections $(29.50 \%)$

2. Enteric infections (14.42\%)

3. Non-communicable digestive diseases (4.77\%)

4. Other non-communicable diseases (4.09\%)

5. Skin infectious diseases (3.59\%)

6. Neonatal disorders (2.96\%)

7. Unknown fever $(2.52 \%)$

8. Other infections $(2.21 \%)$

9. Hemoglobinopathies and hemolytic anemia (1.75\%)

10. Hand, foot and mouth disease (1.59\%)

11. Unintentional injuries (1.58\%)

12. Congenital birth defects (1.21\%)

13. Convulsion and epilepsy (1.05\%)

14. Sepsis $(0.98 \%)$

15. Headache disorders $(0.85 \%)$

16. Cardiovascular diseases $(0.75 \%)$

17. Diabetes and chronic kidney diseases $(0.61 \%)$

18. Nutritional deficiencies $(0.51 \%)$

19. Neurological disorders $(0.30 \%)$

20. Meningitis and encephalitis $(0.27 \%)$

21. Mental disorders (0.26\%)

22. Asthma (0.23\%)

23. Food and drug poisoning $(0.22 \%)$

24. Cirrhosis and other chronic liver diseases $(0.20 \%)$

25. Influenza (0.19\%)

26. Leukemia $(0.19 \%)$

27. Viral hepatitis $(0.17 \%)$

28. HIV and tuberculosis $(0.02 \%)$
2019

1. Lower respiratory tract infections (35.91\%)

2. Influenza (14.23\%)

3. Enteric infections (13.01\%)

4. Non-communicable digestive diseases (4.23\%)

5. Other non-communicable diseases $(3.46 \%)$

6. Skin infectious diseases (3.43\%)

7. Unknown fever (2.95\%)

8. Other infections $(2.52 \%)$

9. Neonatal disorders $(2.44 \%)$

10. Unintentional injuries (2.36\%)

11. Hand, foot and mouth disease (1.70\%)

12. Sepsis (1.65\%)

13. Congenital birth defects (1.17\%)

14. Hemoglobinopathies and hemolytic anemia (1.13\%)

15. Diabetes and chronic kidney diseases (1.04\%)

16. Cardiovascular diseases $(0.86 \%)$

17. Asthma (0.81\%)

18. Convulsion and epilepsy $(0.80 \%)$

19. Neurological disorders $(0.30 \%)$

20. Viral hepatitis $(0.57 \%)$

21. Headache disorders $(0.51 \%)$

22. Mental disorders $(0.43 \%)$

23. Food and drug poisoning $(0.27 \%)$

24. Nutritional deficiencies $(0.26 \%)$

25. Meningitis and encephalitis $(0.10 \%)$

26. HIV and tuberculosis $(0.09 \%)$

27. Cirrhosis and other chronic liver diseases $(0.09 \%$

28. Leukemia $(0.07 \%)$

Figure 3 Morbidities (diagnosis at discharge) among hospitalised children at the Thai Binh Hospital in 2019 compared to 2015 (orange: infectious diseases, white: non-communicable diseases, solid line: stability or increase overtime, dashed line: decrease overtime).

$(120 / 113,999)$ were discharged against medical advice and $0.1 \%$ $(156 / 113,999)$ died. Deaths mainly occurred in children under the age of $5(94.2 \%(147 / 156))$ and in residents from rural areas $(93.0 \%(145 / 156))$. Table 2 shows the cause of death. ID accounted for $40.4 \%$ of cause of deaths and neonatal disorders accounted for $34.6 \%$. LRTI and sepsis were responsible for $16.0 \%$ and $11.5 \%$ of deaths, respectively. Most patients who died resided in rural areas.

\section{DISCUSSION}

This descriptive study was conducted at a provincial paediatric hospital which has limited diagnostic tools. Our main results are as follows: (i) $84 \%$ of hospitalised children were aged under the age of 5; (ii) $61 \%$ of them were diagnosed with an infection, with the four most prevalent conditions being LRTI, influenza, gastrointestinal infections and fever of unknown origin; (iii) $81 \%$ of children were prescribed antibiotics during their stay, whatever the diagnosis; (iv) the mortality rate was about $0.1 \%$ with ID as the leading cause of death.

The high percentage of infants and young children may be explained by the vulnerability of this age group. The anatomical characteristics and immunological immaturity of younger children put them at a higher risk of getting ill in general and of presenting severe forms of ID [11]. Several studies conducted in other countries 
Table 2 Demographics and etiology among 156 children who died at the Thai Binh Paediatric Hospital between 2015 and 2019

\begin{tabular}{lcc}
\hline Mortality & $\boldsymbol{n}$ & Percentage (\%) \\
\hline Under five years old & 147 & 94.2 \\
Male gender & 101 & 64.7 \\
Residence in rural area & 145 & 93.0 \\
Cause of death (diagnosis at discharge) & & \\
Neonatal disorders & 54 & 34.6 \\
Lower respiratory tract infections $^{*}$ & 25 & 16.0 \\
Cardiovascular diseases $^{*}$ & 23 & 14.7 \\
Sepsis $^{*}$ & 18 & 11.5 \\
Fever of unknown origin $^{*}$ & 18 & 11.5 \\
Asthma and other chronic respiratory diseases & 7 & 4.5 \\
Congenital birth defects $^{*}$ & 3 & 1.9 \\
Gastrointestinal infections $^{*}$ & 2 & 1.3 \\
Other non-communicable diseases $_{\text {Cirrhosis and other chronic liver diseases }}$ & 2 & 1.3 \\
Leukaemia $_{\text {Non-communicable gastrointestinal diseases }}$ & 1 & 0.64 \\
Unintentional injuries $^{*}$ & 1 & 0.64 \\
\hline
\end{tabular}

"Infectious diseases.

also reported that the majority of hospitalisations in paediatrics were in children in this age group [11-15], with prevalence ranging from $73 \%$ to $90 \%$. Children below the age of 5 also presented with a higher incidence of neonatal disorders and ID [11,12]. IDs are a leading cause of morbidity and mortality in young children [16]. An estimated 6.2 million children under the age of 15 died in 2018, mostly from preventable causes, and most of whom lived in middle- and low-income countries [1]. In Vietnam, an 8-year retrospective study (2007-2014) among 212,216 hospitalised children under the age of 17 in Hanoi [17] showed that ID was responsible for the majority hospitalisations with RTIs accounting for $37.7 \%$ of cases, other bacterial and parasitic infections for $19.8 \%$, and gastrointestinal disorders for $10.2 \%$.

We observed a high rate of LRTI and influenza in hospitalised children. Previous studies in Vietnam showed that respiratory syncytial virus, enterovirus/rhinovirus, parainfluenza virus and adenovirus were frequently associated with LRTI [18-23]. Bacteria classically described to cause community-acquired pneumonia in Vietnamese children include Streptococcus pneumoniae, Hib and Staphylococcus aureus [24]. The conjugated Hib vaccine was introduced in the expanded immunisation programme in 2006 and the prevalence of Hib has subsequently declined dramatically [24]. Vaccination against invasive pneumococcal diseases, by contrast, is not covered by social security health insurance in Vietnam. In our study, the prevalence of atypical pneumonia was not available. A previous study on 722 hospitalised Vietnamese children [25], showed that atypical pathogens were identified in $29.8 \%$ of cases, mainly Mycoplasma pneumoniae (26.3\%). Viral and virus-bacteria co-infections were frequently detected in children with LRTI [18-21,25].

The decrease in newly diagnosed cases of tuberculosis may reflect a decline in tuberculosis incidence in Vietnam, as a result of several programmatic improvements that have been made, such as vaccination. The significant increase in the prevalence of influenza seen in 2019 may be due to annual variation in influenza incidence but is most likely related to a better availability of influenza-specific PCR. This suggests that laboratory facilities not only play an important role in the definitive and differential diagnosis of ID, but also helps in identifying the true pattern of diseases on which to base priorities in terms of therapeutic needs in the hospital.

In diarrhoeal Vietnamese children, rotavirus was the pathogen most commonly detected with a prevalence of 50.0\% [26-28]. The cost of the rotavirus vaccine is not covered by Vietnamese government. It is expensive and access is difficult for children living in rural areas. Furthermore, norovirus, diarrhoeagenic Escherichia coli, Salmonella spp. and Shigella spp. were also frequently associated with diarrhoea in Vietnamese children [26-28]. Risk factors for diarrhoea in Vietnamese children include, as in many settings, male gender, under the age of 2 , and poor socioeconomic indicators such as household overcrowding and poor hygienic conditions [27]. Improvements in sanitation, in food and water quality are likely to alter the incidence of rotavirus diarrhoea in small children. Moreover, the rotavirus vaccine is targeted for introduction into the expanded immunisation programme to prevent disease [29].

World-wide, malaria is one of the most frequent IDs in children [30]. In our study, no case of malaria was reported over the 5-year period of inclusion. In Vietnam, malaria incidence dramatically decreased by $94.9 \%$ from 1992 to 2014 thanks to the widespread use of artemisinin derivatives and impregnated mosquito nets [31]. No cases of malaria were identified in Thai Binh in 2014 [31].

Antibiotic overuse is an important contributor to the development of antibiotic resistance world-wide. In low-income countries or poor settings, antibiotic prescription is particularly high, because of the fear of bacterial systemic infections when faced with non-specific febrile signs [32]. In a systematic review, Donà et al. [33] showed that between $20 \%$ and $50 \%$ of antibiotic prescriptions in children are potentially unnecessary or inappropriate. Many children still receive broad-spectrum antibiotics for viral infections or antibiotic courses that are significantly longer than needed [33,34]. Hoa et al. conducted a study about knowledge of antibiotics and reported the practices of 96 healthcare providers regarding antibiotic use for respiratory infections among children. Only 19\% had overall knowledge in line with recommended guidelines, and antibiotics were often prescribed or dispensed to treat common colds [35].

The overall hospital mortality rate was lower than reported in other Vietnamese studies. A retrospective survey of 12,389 paediatric emergency visits showed that mortality was $1.1 \%$ [17]. An $8.6 \%$ mortality rate was reported in neonates [36]. The lower mortality rate observed at the Thai Binh Paediatric Hospital may be explained by the referral of more severe cases to the National Hospital of Paediatrics, in Hanoi (12.0\%). Moreover, due to cultural habits, some Vietnamese people do not want their family members to die at the hospital but at home, some parents of seriously ill children $(0.1 \%)$ applied for hospital discharge against medical advice. Mortality data for these patients were not available. Furthermore, most children who died came from rural areas, suggesting that they may not have had easy and early access to hospital treatment.

Our study has some limitations. The study was retrospective and lack microbiological documentation in most cases of infections. The study did not capture information about socioeconomic conditions, environmental factors and living conditions at home. Furthermore, the time between onset of symptoms and hospitalisation was not available. Finally, no distinction was made between initial visits and readmission of the same patients. Nevertheless, this data serves as a basis for the identification of needs for 
diagnostic tools and for future evaluation of the effect of targeted implementations of such facilities. The data also helps in designing standardised protocols for the proper management of the most common diseases presenting in this hospital and to strategically provide the necessary equipment for child healthcare in economically limited conditions. Further studies aimed at identifying the pathogens frequently responsible for infections in children hospitalised at the Thai Binh hospital should be conducted. Based on these results, point-of-care tests, including real-time PCR assays to identify common pathogens should be implemented for more accurate diagnosis and more appropriate antibiotic use.

\section{CONFLICTS OF INTEREST}

The authors declare they have no conflicts of interest.

\section{AUTHORS' CONTRIBUTION}

TDP, VTH, TLD and PG contributed to the experimental design. TDP, VTH, TLD, XDT, DLP, MMT, VND, VKD, TTD, NTN, TTV, TLV and TMCN collected the data. TDP, VTH, TLD, XDT, DLP, MMT, VND, VKD, TTD, TTV, TLV and TMCN analysed the data. TDP, VTH, TLD, PM and PG contributed to interpretation and writing. DTN, NTN, DCN, NTH, TLV, TMCN and PM contributed to critically reviewing the manuscript. TDP and VTH contributed equal work PG coordinated the work.

\section{FUNDING}

No financial support was provided.

\section{REFERENCES}

[1] World Health Organization (WHO). Children: reducing mortality. Available from: https://www.who.int/news-room/fact-sheets/ detail/children-reducing-mortality (accessed May 3, 2020).

[2] George IO, Alex-Hart BA, Frank-Briggs AI. Mortality pattern in children: a hospital based study in Nigeria. Int J Biomed Sci 2009;5;369-72.

[3] General Statistics office Viet Nam. Viet Nam Multiple Indicator Cluster Survey. Final Report. Ha Noi, Viet Nam; 2014. Available from: https://www.unicef.org/vietnam/sites/unicef.org.vietnam/files/ 2018-07/MICS_VIET_NAM_2014_ENG_310815.pdf (accessed May 3, 2020).

[4] Institute for Health Metrics and Evaluation. GDB compare. Available from: https://vizhub.healthdata.org/gbd-compare/?fbclid=IwAR3d9KQFluz4KLccj9A80HtWpjudNriWlhl1qF_s0cPbB4j1m2R_UPauKgo (accessed May 3, 2020).

[5] Nguyen KV, Thi Do NT, Chandna A, Nguyen TV, Pham CV, Doan PM, et al. Antibiotic use and resistance in emerging economies: a situation analysis for Viet Nam. BMC Public Health 2013;13;1158.

[6] Peters L, Olson L, Khu DTK, Linnros S, Le NK, Hanberger H, et al. Multiple antibiotic resistance as a risk factor for mortality and prolonged hospital stay: a cohort study among neonatal intensive care patients with hospital-acquired infections caused by gram-negative bacteria in Vietnam. PLoS One 2019;14;e0215666.
[7] Wikipedia. Thái Bình Province. Wikipedia. Available from: https://en.wikipedia.org/wiki/Thái_Bình_Province (accessed May 3, 2020).

[8] City Population. Thái Bình Province in Vietnam. Available from: https://www.citypopulation.de/en/vietnam/prov/admin/ (accessed May 3, 2020).

[9] Hoang VT, Dao TL, Minodier P, Nguyen DC, Hoang NT, Dang $\mathrm{VN}$, et al. Risk factors for severe pneumonia according to WHO 2005 criteria definition among children $<5$ years of age in Thai Binh, Vietnam: a case-control study. J Epidemiol Glob Health 2019;9;274-80.

[10] World Health Organization (WHO). ICD-10 version: 2015. Available from: https://icd.who.int/browse10/2015/en\#/ (accessed May 3, 2020).

[11] Ferrer APS, Sucupira ACSL, Grisi SJFE. Causes of hospitalization among children ages zero to nine years old in the city of São Paulo, Brazil. Clinics (São Paulo) 2010;65;35-44.

[12] Leyenaar JK, Ralston SL, Shieh MS, Pekow PS, Mangione-Smith R, Lindenauer PK. Epidemiology of pediatric hospitalizations at general hospitals and freestanding children's hospitals in the United States. J Hosp Med 2016;11;743-9.

[13] Witt WP, Weiss AJ, Elixhauser A. Overview of hospital stays for children in the United States, 2012: Statistical Brief \#187. Healthcare Cost and Utilization Project (HCUP) Statistical Briefs [Internet]. Rockville, MD: Agency for Healthcare Research and Quality (US); 2006.

[14] Monteiro LGS, Chauque A, Barros MP, Ira TR. Determinants of antibiotic prescription in paediatric patients: the case of two hospitals in Maputo, Mozambique. South Afr J Child Health 2017;11;109-11.

[15] Nguyen TKP, Nguyen DV, Truong TNH, Tran MD, Graham SM, Marais BJ. Disease spectrum and management of children admitted with acute respiratory infection in Viet Nam. Trop Med Int Health 2017;22;688-95.

[16] Global Burden of Disease Child and Adolescent Health Collaboration, Kassebaum N, Kyu HH, Zoeckler L, Olsen HE, Thomas K, et al. Child and adolescent health from 1990 to 2015: findings from the global burden of diseases, injuries, and risk factors 2015 study. JAMA Pediatr 2017;171;573-92.

[17] Nguyen NTT, Dien TM, Schindler C, Lien NTB, Probst-Hensch N, Lan VTH, et al. Childhood hospitalisation and related deaths in Hanoi, Vietnam: a tertiary hospital database analysis from 2007 to 2014. BMJ Open 2017;7;e015260.

[18] Pham HT, Nguyen PTT, Tran ST, Phung TTB. Clinical and pathogenic characteristics of lower respiratory tract infection treated at the Vietnam National Children's Hospital. Can J Infect Dis Med Microbiol 2020;2020;7931950.

[19] Tran DN, Trinh QD, Pham NTK, Vu MP, Ha MT, Nguyen TQN, et al. Clinical and epidemiological characteristics of acute respiratory virus infections in Vietnamese children. Epidemiol Infect 2016;144;527-36.

[20] Do AHL, van Doorn HR, Nghiem MN, Bryant JE, Hoang THt, Do $\mathrm{QH}$, et al. Viral etiologies of acute respiratory infections among hospitalized Vietnamese children in Ho Chi Minh City, 2004-2008. PLoS One 2011;6;e18176.

[21] Alroy KA, Do TT, Tran PD, Dang TQ, Vu LN, Le NTH, et al. Expanding severe acute respiratory infection (SARI) surveillance beyond influenza: the process and data from 1 year of implementation in Vietnam. Influenza Other Respir Viruses $2018 ; 12 ; 632-42$. 
[22] Althouse BM, Flasche S, Minh LN, Thiem VD, Hashizume M, Ariyoshi K, et al. Seasonality of respiratory viruses causing hospitalizations for acute respiratory infections in children in Nha Trang, Vietnam. Int J Infect Dis 2018;75;18-25.

[23] Yoshihara K, Le MN, Toizumi M, Nguyen HA, Vo HM, Odagiri $\mathrm{T}$, et al. Influenza B associated paediatric acute respiratory infection hospitalization in central Vietnam. Influenza Other Respir Viruses 2019;13;248-61.

[24] Phuong NTK, Hoang TT, Van PH, Tu L, Graham SM, Marais BJ. Encouraging rational antibiotic use in childhood pneumonia: a focus on Vietnam and the Western Pacific region. Pneumonia (Nathan) 2017;9;7.

[25] Huong PLT, Hien PT, Lan NTP, Binh TQ, Tuan DM, Anh DD. First report on prevalence and risk factors of severe atypical pneumonia in Vietnamese children aged 1-15 years. BMC Public Health 2014;14;1304.

[26] Nguyen TV, Van PL, Huy CL, Gia KN, Weintraub A. Etiology and epidemiology of diarrhea in children in Hanoi, Vietnam. Int J Infect Dis 2006;10;298-308.

[27] Anders KL, Thompson CN, Van Thuy NT, Nguyet NM, Tu LTP, Dung TTN, et al. The epidemiology and aetiology of diarrhoeal disease in infancy in southern Vietnam: a birth cohort study. Int J Infect Dis 2015;35;3-10.

[28] Hien BTT, Trang DT, Scheutz F, Cam PD, Mølbak K, Dalsgaard A. Diarrhoeagenic Escherichia coli and other causes of childhood diarrhoea: a case-control study in children living in a wastewater-use area in Hanoi, Vietnam. J Med Microbiol 2007;56;1086-96.
[29] Tu HAT, Rozenbaum MH, Coyte PC, Li SC, Woerdenbag HJ, Postma MJ. Health economics of rotavirus immunization in Vietnam: potentials for favorable cost-effectiveness in developing countries. Vaccine 2012;30;1521-8.

[30] World Health Organization (WHO). World malaria report 2018. Available from: https://apps.who.int/iris/bitstream/handle/10665 /275867/9789241565653-eng.pdf?ua=1 (accessed May 3, 2020).

[31] Goldlust SM, Thuan PD, Giang DDH, Thang ND, Thwaites GE, Farrar J, et al. The decline of malaria in Vietnam, 1991-2014. Malar J 2018;17;226.

[32] Koopmans LR, Finlayson H, Whitelaw A, Decloedt EH, Dramowski A. Paediatric antimicrobial use at a South African hospital. Int J Infect Dis 2018;74;16-23.

[33] Donà D, Barbieri E, Daverio M, Lundin R, Giaquinto C, Zaoutis $\mathrm{T}$, et al. Implementation and impact of pediatric antimicrobial stewardship programs: a systematic scoping review. Antimicrob Resist Infect Control 2020;9;3.

[34] Nguyen PT, Tran HT, Fitzgerald DA, Graham SM, Marais BJ. Antibiotic use in children hospitalised with pneumonia in Central Vietnam. Arch Dis Child 2020;105;713-19.

[35] Hoa NQ, Larson M, Chuc NTK, Eriksson B, Trung NV, Stålsby CL. Antibiotics and paediatric acute respiratory infections in rural Vietnam: health-care providers' knowledge, practical competence and reported practice. Trop Med Int Health 2009; $14 ; 546-55$.

[36] Tran HT, Doyle LW, Lee KJ, Dang NM, Graham SM. Morbidity and mortality in hospitalised neonates in central Vietnam. Acta Paediatr 2015;104;e200-e5. 